\title{
Multilayer Machine Learning-Assisted Optimization-Based Robust Design and Its Applications to Antennas and Arrays
}

\author{
Qi Wu, Weiqi Chen, Chen Yu, Haiming Wang, and Wei Hong
}

\begin{abstract}
An efficient multilayer machine learning-assisted optimization (ML-MLAO)-based robust design method is proposed for antenna and array applications. Machine learning methods are introduced into multiple layers of the robust design process, including worst-case analysis (WCA), maximum input tolerance hypervolume (MITH) searching, and robust optimization, considerably accelerating the whole robust design process. First, based on a surrogate model mapping between the design parameters and performance, WCA is performed using a genetic algorithm to ensure reliability. MITH searching is then carried out using a double-layer MLAO (DL-MLAO) framework to find the MITH of the given design point. Next, based on the training set obtained using DLMLAO, correlations between the design parameters and the MITH are learned. The robust design is carried out using surrogate models for both the performance and the MITH, and these models are updated online following the ML-MLAO scheme. Furthermore, two examples, including an array synthesis problem and an antenna design problem, are used to verify the proposed ML-MLAO method. Finally, the numerical results and computation time are discussed to demonstrate the effectiveness of the proposed method.
\end{abstract}

Index Terms-Antennas, antenna tolerance analysis, arrays, optimization methods

\section{INTRODUCTION}

$\mathbf{R}$ OBUST design is one of the most crucial aspects in the design of modern antennas and arrays, which aims to learn the correlation between the input and output tolerance, thereby finding a balance between the robustness and performance of the final design and providing guidelines for the integration and manufacturing process [1]-[5]. With the rapid development of commercial full-wave electromagnetic (EM) simulation tools, robust design approaches independent of the computational EM process have been widely investigated with optimization schemes [3], [4], [6] and surrogateassisted modeling approaches [2], [7].

Robust design methods can be generally divided into separate categories to resolve two issues: 1) methods employed to find the output tolerance when the input tolerance is known and 2) methods used to find the input tolerance when the output tolerance is known. Worstcase analysis (WCA) was introduced to resolve issue 1, which aims to find the scenario with the worst-case performance (WCP) within the given input tolerance on the nominal solution [4], [8]. Issue 2 involves a more rigorous process based on the reliable results for issue 1. A popular approach is maximum input tolerance hypervolume (MITH) searching, which first defines a hypervolume representing the input tolerance and then finds the MITH that satisfies the predefined output

\footnotetext{
Manuscript received July 17, 2020; revised

Date of publication ; date of current

; accepted

. This work was supported in part by the National Key R\&D Program of China under Grant 2020YFB1804901, the National Natural Science Foundation of China under Grant 61960206006, the Science Foundation of Jiangsu Province of China under Grant BK20191011, and the Fundamental Research Funds for the Central Universities of China under Grants 2242020K40112 and 2205020520223.

The authors are with the State Key Laboratory of Millimeter Waves, Southeast University, Nanjing 210096, China, and with Purple Mountain Laboratories, Nanjing 211111, China (e-mail: qiwu@seu.edu.cn; wq_chen@seu.edu.cn; chenyu@seu.edu.cn; hmwang@seu.edu.cn; weihong@seu.edu.cn). (Corresponding author: Haiming Wang.)

Color versions of one or more of the figures in this paper are available online at http://ieeexplore.ieee.org.

Digital Object Identifier
}

tolerance. In [4], a novel hybrid strategy consisting of particle swarm optimization for WCA and iterative input tolerance hypervolume (ITH) shrinking was used to find the MITH, but the ratios between different design parameters must be decided in advance and cannot be changed during the iterative process. In [2], a novel sampling strategy (SS) is introduced to implement efficient MITH searching. SS can automatically find the quasi-optimal shape of the MITH efficiently. However, the fidelity of the MITH is dependent on the sampling points in every iteration, and there is no guarantee that the real WCPs of the obtained MITH are found during the process, which leads to possible bias in the calculated MITH.

Many surrogate model-based optimization methods have been introduced to address EM problems [9]-[16]. The key motivation of the surrogate model-based optimization algorithm is to use efficient surrogate models to accelerate the optimization procedure; these surrogate models can be built using physically coarse models or datadriven modeling strategies. Various ML methods, such as Gaussian process regression (GPR) [9], [13] and artificial neural networks (ANNs) [14], have been introduced to build the surrogate models of EM components and applied to machine learning-assisted optimization (MLAO) schemes. In [7], the surrogate model is combined with a genetic algorithm (GA) to find the WCP when the input tolerance is known. In [2], surrogate models have been applied in MITH searching. However, the surrogate models have been used to replace only full-wave EM simulations; consequently, the large number of function calls required in the robust design process will still result in a high time consumption.

In this Communication, based on multilayer machine learningassisted optimization (ML-MLAO), an efficient and reliable robust design scheme is proposed and applied to antennas and arrays. To the authors' best knowledge, this is the first time that the utilization scope of MLAO algorithm has been expanded to the prediction and optimization of not only antenna and array responses, but also MITHs and robust designs, therefore upgrade the reliability of the evaluated MITH, and the efficiency when compared with conventional optimization-based robust design algorithms. A novel double-layer MLAO (DL-MLAO) method for efficient and reliable MITH searching is introduced in Section II. Section III integrates the proposed DL-MLAO method into ML-MLAO to achieve robust antenna design. Two examples, array synthesis and antenna design problems, are used to verify the proposed method in Section IV. Finally, Section V concludes this work.

\section{Worst-CASE ANAlysis AND MAXIMUM InPUT TOLERANCE HYPERVOLUME SEARCHING}

\section{A. Mathematical Formulation of the Problems}

Consider a set of $Q$ nonlinear differentiable functions, each with $P$ variables: $y_{q}(\boldsymbol{x})=y_{q}\left(x_{1}, x_{2}, \ldots, x_{P}\right), \quad q=1,2, \ldots, Q$. The uncertainties of the input parameters are defined by the input tolerances on the variables: $\boldsymbol{\delta} \triangleq\left[\delta_{1}, \delta_{2}, \ldots, \delta_{P}\right]^{\mathrm{T}}, \quad \delta_{p} \geq 0$. Therefore, the input tolerance interval is represented as

$$
\omega_{\boldsymbol{x}, \boldsymbol{\delta}} \triangleq\{\boldsymbol{t} \mid(\boldsymbol{x}-\boldsymbol{\delta} \leq \boldsymbol{t} \leq \boldsymbol{x}+\boldsymbol{\delta})\}
$$




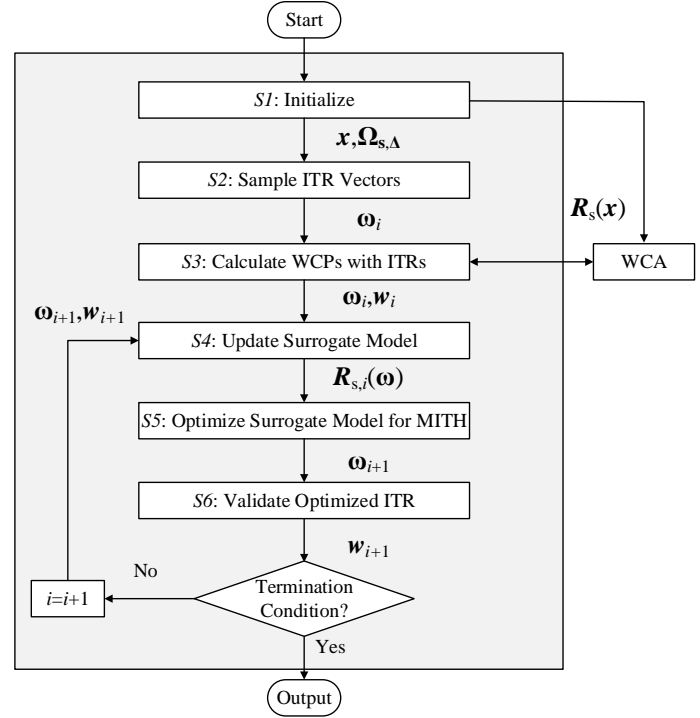

Fig. 1. Flow diagram of the DL-MLAO algorithm for MITH searching.

where $t$ represents possible parameter values. The WCP is defined as the specific parameter $\hat{\boldsymbol{x}}$ that causes the maximum deviation; i.e.,

$$
F_{q}(\hat{\boldsymbol{x}}) \triangleq \max _{\hat{\boldsymbol{x}} \in \omega_{\boldsymbol{x}, \boldsymbol{\delta}}} f_{q}(\hat{\boldsymbol{x}}) .
$$

For $Q>1$, there may exist multiple WCP points within the design space. The collection of WCPs is defined as $\boldsymbol{F}(\boldsymbol{x})$. The methods employed to search for the WCP represent the infrastructure of robust antenna design. Within a given input tolerance region (ITR), a global optimization method is normally required over the tolerance interval to search for reliable WCP points [8].

The uncertainties in the design parameters of a nominal point $\boldsymbol{x}$, known as the ITR, can be modeled as a hyperrectangle defined by $\boldsymbol{\delta}(\boldsymbol{x})$. The ITH can be defined to evaluate the size of the ITR, which can be represented as the product of the uncertainties:

$$
T_{\text {ITR,peak }}(\boldsymbol{x})=\prod_{p=1}^{P} \boldsymbol{\delta}(\boldsymbol{x}) .
$$

A more important issue in robust antenna design is to search for the maximum $T_{\mathrm{ITH}}$ if the output tolerance region (OTR) is known, which can be regarded as an optimization problem if WCA can be performed with high accuracy. Similar to the ITR, the OTR can be defined as $\boldsymbol{\Delta} \triangleq\left[\Delta_{1}, \Delta_{2}, \ldots, \Delta_{Q}\right]^{\mathrm{T}}, \quad \Delta_{q} \geq 0$. The output tolerance interval can be represented as

$$
\Omega_{\boldsymbol{s}, \boldsymbol{\Delta}} \triangleq\{\boldsymbol{y} \mid(\boldsymbol{y}-\boldsymbol{\Delta} \leq \boldsymbol{s} \leq \boldsymbol{y}+\boldsymbol{\Delta})\},
$$

where $\boldsymbol{s}=\left[s_{1}, s_{2}, \ldots, s_{Q}\right]^{\mathrm{T}}$ represents possible function values. The MITH searching can be numerically represented as

$$
\max T_{\mathrm{ITH}}(\boldsymbol{x}), \text { s.t. } \boldsymbol{F}(\boldsymbol{x}) \in \Omega_{\boldsymbol{s}, \boldsymbol{\Delta}} .
$$

\section{B. Maximum Input Tolerance Hypervolume Searching}

By introducing MLAO methods, the WCP of a given nominal solution and its ITR can be efficiently located with the help of a lowcomplexity surrogate model [7]. First, design vectors in the given ITR are sampled, and the calculation is performed using HF simulations. Second, surrogate models are built using ML methods for each design objective. Third, the WCPs are searched using populationbased optimization methods and surrogate models. Note that for cases such as array synthesis, if HF responses can be generated with a negligible time cost, then HF models can also be utilized directly for population-based optimization procedures. The typical computation time for WCA is approximately $5 \mathrm{~s}$ if surrogate models (for antenna design) or HF models (for array synthesis) are used to produce the required responses.

Once an accurate WCP can be acquired for any given nominal point and its corresponding ITR, the MITH searching problem can be simplified to a constraint optimization problem. For a given design point, the optimization objective is to find the ITR corresponding to the MITH among all the possible ITRs that fulfill the OTR. In most practical problems, the ratio between the different dimensions of the optimal ITR is unknown. For such problems, populationbased optimization methods (rather than traditional gradient-based local-search optimization methods) are some of the best options for yielding robust, globally optimized solutions. However, the time cost issue arises when considering the computation time for WCA and the large number of function calls required by population-based optimization methods. For a three-parameter robust antenna design problem, a population number of 50 and an iteration number of 100 are often required, resulting in a computation time of approximately $7 \mathrm{~h}$ for one MITH search process, which is unacceptable for future robust optimization.

Here, a DL-MLAO algorithm is proposed to accelerate the MITH search process. The workflow of the proposed DL-MLAO algorithm is illustrated in Fig. 1. The detailed steps of the DL-MLAO MITH search procedure are summarized as follows:

Step 1. Define the design point, design space, OTR and utilized model: The design space must be set larger than the design space in the previous optimization procedure to handle all possible situations where the design points are located along the boundaries. The OTR for antenna design can be set for the worst reflection coefficient within the bandwidth, sidelobe level (SLL), gain and others. The utilized model can be a surrogate model for HF EM simulations or other computationally inexpensive models.

Step 2. Sample ITR vectors in the design space: Using sampling strategies such as Latin hypercube sampling (LHS), a number $N_{\mathrm{r}}$ of ITR vectors are sampled within the design space. Considering the differences in the sensitivities of different design parameters, random sampling strategies are essential for exploring the whole design space. Uniform sampling strategies, including the sampling of $N_{\mathrm{u}}+1$ initial design points, or sampling with constraints can also be incorporated to accelerate the algorithm if some prior knowledge about the MITH is known.

Step 3. Simulate the WCP responses for given ITR vectors: Following the WCA method proposed above, the WCP responses for the sampled ITH vectors are simulated. The ITR vectors $\boldsymbol{\omega}_{\mathrm{i}}$ and their corresponding WCP responses $\boldsymbol{w}_{\text {i }}$ are combined to establish the dataset. For a number $Q$ of design objectives, WCA is performed $Q$ times. Therefore, while the WCP responses are identical for one given nominal point, there may exist a number $N_{\mathrm{WCP}} \leq Q$ of several corresponding $\boldsymbol{x}_{\mathrm{WCP}}$ that perform the worst for different design objectives.

Step 4. Train the surrogate models: Here, due to the small size of the dataset, the single-output Gaussian process regression (SOGPR) method is introduced to train the surrogate models [17]. The sampled ITR vectors $\boldsymbol{\omega}_{\mathrm{i}}$ are regarded as input variables $\boldsymbol{X}$, and the corresponding WCP responses $\boldsymbol{w}_{\mathrm{i}}$ are regarded as output variables $\boldsymbol{y}$. In this step, $Q$ surrogate models for the WCP points are built based on the established dataset.

Step 5. Optimize for the MITH: Here, the fitness function $f_{\mathrm{MITH}}$ is defined as follows:

$$
f_{\mathrm{MITH}}=\left\{\begin{array}{c}
D\left(\boldsymbol{F}(\boldsymbol{x}), \Omega_{\boldsymbol{s}, \boldsymbol{\Delta}}\right), \boldsymbol{F}(\boldsymbol{x}) \notin \Omega_{\boldsymbol{s}, \boldsymbol{\Delta}}, \\
-T_{\mathrm{ITH}, \text { peak }}(\boldsymbol{x}), \boldsymbol{F}(\boldsymbol{x}) \in \Omega_{\boldsymbol{s}, \boldsymbol{\Delta}},
\end{array}\right.
$$




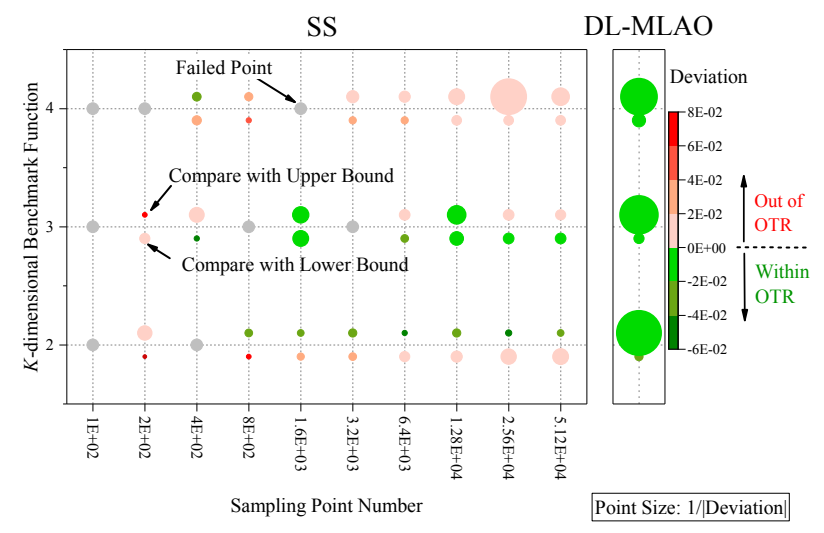

Fig. 2. Performance comparison between SS and the proposed DL-MLAO.

where $D\left(\boldsymbol{F}(\boldsymbol{x}), \Omega_{\boldsymbol{s}, \boldsymbol{\Delta}}\right)$ represents the distance between the WCP and the OTR,

$$
\begin{gathered}
D\left(\boldsymbol{F}(\boldsymbol{x}), \Omega_{\boldsymbol{s}, \boldsymbol{\Delta}}\right)=\sum_{q=1}^{Q} \max \left\{\operatorname { m i n } \left\{\left|\max \left\{f_{q}(\boldsymbol{t})\right\}-\left(y_{q}+\Delta_{q}\right)\right|,\right.\right. \\
\left.\left.\left|\max \left\{f_{q}(\boldsymbol{t})\right\}-\left(y_{q}-\Delta_{q}\right)\right|\right\}, 0\right\} .
\end{gathered}
$$

For cases in which $\boldsymbol{F}(\boldsymbol{x}) \notin \Omega_{\boldsymbol{s}, \boldsymbol{\Delta}}, f_{\mathrm{MITH}}$ will always be positive but will decrease when the predicted WCP approaches the OTR. For cases in which $\boldsymbol{F}(\boldsymbol{x}) \in \Omega_{\boldsymbol{s}, \boldsymbol{\Delta}}, f_{\mathrm{MITH}}$ will be negative and will decrease when $T_{\text {ITH,peak }}$ increases. The proposed fitness function offers a continuous optimization objective space and can find the MITH efficiently.

Step 6. Validate the predicted MITH and update the database: The MITH predicted by the above optimization procedure is then validated by the accurate WCA method. The termination criterion, such as the maximum number of iterations $N_{\text {iter }}$ or the maximum number of available results $N_{\text {num }}$, is then checked. If no criterion is met, the surrogate model for the WCP prediction is updated online, and then, Step 4 is repeated.

The DL-MLAO MITH search method is a nested loop structure that uses WCA as the HF simulation for the MITH search process. In [2], a novel MITH search method called SS is introduced. SS can automatically find the quasi-optimal shape of the MITH efficiently by iteratively sampling random points within the design space. For practical problems, $N_{\mathrm{s}}=7500$ sampling points and $N_{\text {iter }}=6$ iterations are suggested. Although SS is very efficient and effective, two issues are worth further consideration: 1) for practical problems with a large design space and no prior knowledge about the MITH dimensions, the MITH may not be found if the number of sampling points is relatively small, and 2) the evaluated MITH is not accurate because the WCP may not be located within the sampling set.

A $K$-dimensional, nonconvex, nonseparable benchmark function Ackley is evaluated to verify the proposed DL-MLAO MITH searching, which is defined by

$$
g(\boldsymbol{x})=\sum_{k=1}^{K-1}\left(e^{-0.2} \sqrt{x_{k}^{2}+x_{k+1}^{2}}+3\left(\cos \left(2 x_{k}\right)+\sin \left(2 x_{k+1}\right)\right)\right) .
$$

The design point is set as $x_{k}=1, k=1, \ldots, K$, with the OTR of $[-1,1]$ around $g(\boldsymbol{x})$. A performance comparison between SS and the proposed DL-MLAO is given in Fig. 2 for different input dimensions and sampling point numbers $N_{\mathrm{s}}$ of SS. After calculating the MITHs using SS and DL-MLAO, the GA is introduced to find the WCPs, which are then compared with the given OTR to validate the MITHs. The deviations are calculated by comparing the WCPs with the upper

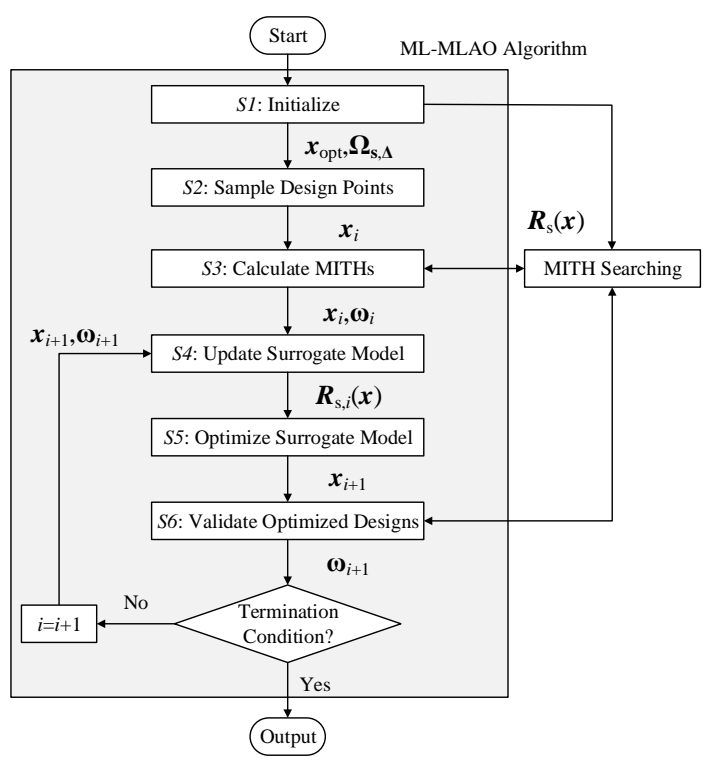

Fig. 3. Flow diagram of the ML-MLAO algorithm for robust optimization.

and lower bounds and are shown using different colors and point sizes.

Fig. 2 reveals several issues. First, due to the limitation of the sampling point number, SS may fail in finding the MITH. Second, in many cases, the WCP of the MITH found by SS is outside the OTR, which means that the true WCPs have not been found when using SS. Third, with increasing input parameter number, the ability of the conventional SS to find the reliable MITH is limited. Compared with SS, the proposed DL-MLAO can obtain MITH results with their WCPs within the OTR but close to the OTR bounds. Considering both efficiency and reliability, the proposed DL-MLAO method can be very helpful for designing practical antennas and arrays.

\section{RoBUST OPTIMIZATION}

Based on the WCA and MITH search algorithms proposed above, robust antenna design can be accomplished using robust optimization for different applications, including array synthesis and antenna design. The ML-MLAO method is proposed based on the MLAO scheme by applying DL-MLAO MITH searching as the HF simulation procedure. The workflow of the ML-MLAO algorithm for robust antenna design is given in Fig. 3. The detailed steps are summarized as follows:

Step 1. Define the initial design points, design constraints, OTR, etc.: The robust design procedure can be implemented after finishing the optimization procedure, from which the number $N_{\text {ini }}$ of initial design points can be obtained. In addition, the design space and constraints should also be defined, as should the OTR.

Step 2. Sample the design points around the initial design points: One practical antenna problem is to find the most robust antenna design based on several optimal design vectors [4]. In this case, the robust design process is implemented based on assumptions that the most robust designs are located around the initial design points obtained by the optimization procedure. Therefore, $k_{d}$ design points around each design point are randomly sampled using strategies such as LHS.

Step 3. Calculate the MITH at every sampling point and establish the database: The MITHs corresponding to every sampled design point, in addition to the initial design points, are calculated using the abovementioned DL-MLAO method.

Step 4. Build surrogate models using the database: Surrogate models are established to build relationships between the locations 


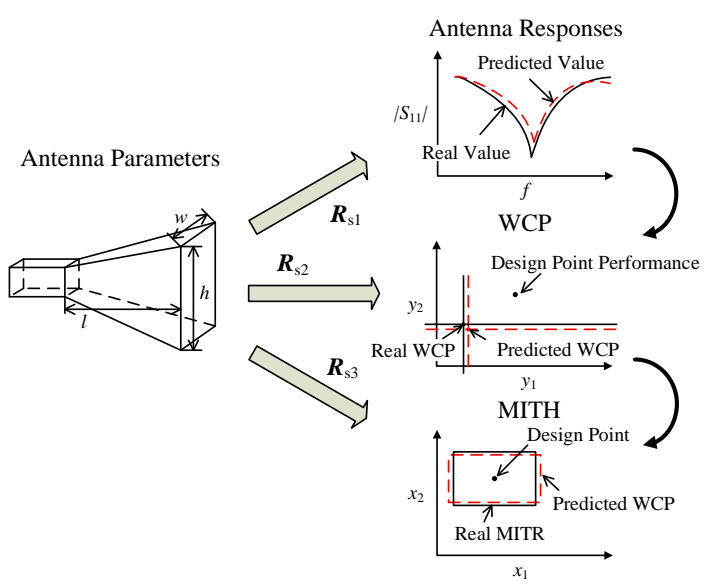

Fig. 4. Surrogate models for antenna responses, WCP points and MITHs.

of the sampling points and the corresponding MITHs. For antenna designs with $N$ parameters, $N$ surrogate models are established to make predictions about the MITHs at new points. Here, the sampled design points $\boldsymbol{x}_{i}$ are regarded as input variables $\boldsymbol{X}$, and the corresponding MITH responses $\omega_{\mathrm{i}}$ are regarded as output variables $\boldsymbol{y}$.

Step 5. Optimize: Here, different optimization methods and different fitness functions can be implemented for different design applications. Different design objectives, such as antenna size, robustness and performance, can be optimized simultaneously using multiobjective optimization methods. Single-objective optimization can also be utilized if robust designs are needed only around the initial design points.

Step 6. Verify the results and update the database: The predicted optimization results of the MITHs are verified using the proposed DL-MLAO method, and the predicted antenna performance is verified using EM simulations. The termination criterion, such as the maximum number of iterations $i_{\mathrm{ML}}$ or the maximum number of unchanged iterations, is then checked. If one of the termination conditions is met, the process is stopped; otherwise, the database is updated, and Step 4 is repeated.

The ML-MLAO method uses the MITH search strategy as the HF simulation procedure in the MLAO scheme. The accuracy and robustness of the results are ensured by the accuracy of DL-MLAO MITH searching. Fig. 4 depicts the operating principle of the proposed ML-MLAO method. Based on the established surrogate model $\boldsymbol{R}_{\mathrm{s} 1}$ between the design parameters and antenna responses, the WCP values of different design points are calculated and utilized to build a surrogate model $\boldsymbol{R}_{\mathrm{s} 2}$ between the design parameters and the WCP. By using the two surrogate models established above, a training set consisting of antenna parameters and MITHs is constructed to build a surrogate model $\boldsymbol{R}_{\mathrm{s} 3}$. The ML-MLAO algorithm offers an efficient solution to build surrogate models, make predictions of new design points and update surrogate models online, thereby efficiently searching for robust design points or Pareto fronts consisting of robust information.

\section{VERIFICATION EXAMPLES}

In this section, an array synthesis problem and an antenna design problem are used to verify the proposed ML-MLAO-based robust design method.

\section{A. Array Synthesis}

Consider a symmetric linear uniformly spaced antenna array consisting of $2 N_{\text {ele }}$ isotropic elements. The element spacing is $d=0.5 \lambda$,
TABLE I

TWO DESIGN POINTS FOR ITERATION NUMBER $n_{a}=10$ BEFORE AND AFTER THE ROBUST DESIGN PROCESS.

\begin{tabular}{cccccccc}
\hline Before & Ele. 1 & Ele. 2 & Ele. 3 & Ele. 4 & Ele. 5 & MITH & SLL \\
\hline 1 & 50.5 & 0.1 & 0.1 & 18.6 & 14.0 & 2891.2 & -14.20 \\
2 & 2.2 & 319.4 & 6.6 & 3.6 & 341.0 & 4162.5 & -14.26 \\
\hline After & Ele. 1 & Ele. 2 & Ele. 3 & Ele. 4 & Ele. 5 & MITH & SLL \\
\hline 1 & 49.5 & 13.1 & 0.1 & 26.0 & 19.3 & 5705.8 & -13.92 \\
2 & 1.9 & 319.5 & 7.1 & 3.2 & 339.0 & 5038.7 & -14.11 \\
\hline
\end{tabular}

The unit for the phase is degrees, the unit for the MITH is degrees ${ }^{6}$, and the unit for the SLL is $\mathrm{dB}$.

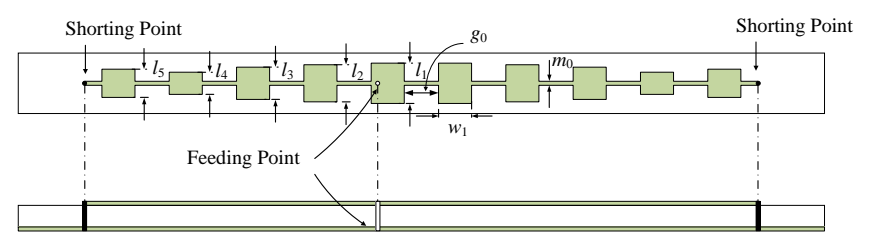

Fig. 5. Structure of the series-fed microstrip antenna array.

and the magnitudes of the elements are uniformly excited. The phases of the elements are optimized to achieve the lowest SLL. Two design results are preliminarily optimized using MLAO optimization methods [18] and then examined and modulated using the ML-MLAO method to obtain robust design points around them. The search range for each design point is $\pm 20^{\circ}$ within the initial designs. The OTR is set as the SLL of $-12 \mathrm{~dB}$. The element phases, MITHs and SLLs before and after the robust design process are given in Table I.

Two designs give similar SLL initially, while design 1 suffers a much lower MITH than design 2, which means that the latter is more robust than the former before the robust design. Upon applying ML-MLAO, the two designs are both modulated to achieve better MITH performance. Although design 1 has a worse SLL performance than design 2 after the robust design, it has a higher MITH value of approximately 5705.8 , which means that design 1 is more robust than design 2 after the robust design process.

\section{B. Antenna Design}

In contrast to the array synthesis problem discussed above, the solution of the antenna design problem relies on the prediction accuracy of the surrogate models trained with the datasets established by full-wave EM simulations. For antennas with a large design parameter range, acquiring accurate surrogate models for the entire design space is difficult if time is limited. Therefore, local surrogate models surrounding the possible optimal design points should be established and updated online during the robust design process rather than surrogate models suitable for the entire design space. The robust antenna design algorithm is divided into two phases: the optimization phase and the robust optimization phase.

In the optimization phase, the MLAO in [18] is applied to calculate the optimal designs under the constraint of forbidden areas by previously optimized design points [4]. By implementing forbidden areas, multiple local optima are obtained with predefined numbers.

In the robust optimization phase, the ML-MLAO algorithm is implemented. Particularly, for multiobjective robust design considering both antenna performance and robustness, the Pareto front $\mathbf{P}_{\text {pre }, i, j}$ and corresponding worst case $\boldsymbol{w}_{i, j}$ are predicted based on the learned correlations, in which $i$ and $j$ represent the indexes of the outer loop and inner loop, respectively. In the inner loop, ML-MLAO is implemented to achieve an accurate Pareto front 




(a)



(b)

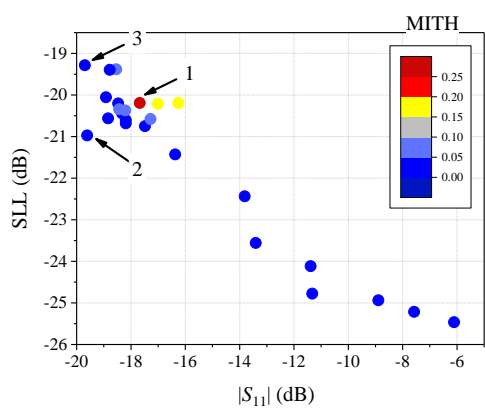

(c)

Fig. 6. Pareto fronts for the designed SMAA after three iterations: (a) $\mathbf{P}_{\text {pre, } 4}$, (b) $\mathbf{P}_{\text {val2,4 }}$ and (c) $\mathbf{P}_{\text {upd,4 }}$.

TABLE II

OPTIMIZED SMAA PARAMETERS OF THE SELECTED DESIGNS.

\begin{tabular}{ccccccccc}
\hline Item & MITH & $\left|S_{11}\right|$ & SLL & $l_{1}(\mathrm{~mm})$ & $l_{2}(\mathrm{~mm})$ & $l_{3}(\mathrm{~mm})$ & $l_{4}(\mathrm{~mm})$ & $l_{5}(\mathrm{~mm})$ \\
\hline 1 & 0.271 & -17.68 & -20.19 & $20.041 \pm 0.616$ & $20.344 \pm 0.863$ & $16.632 \pm 0.853$ & $12.233 \pm 0.907$ & $10.855 \pm 0.658$ \\
2 & 0.011 & -19.61 & -20.97 & $19.671 \pm 0.296$ & $21.000 \pm 0.279$ & $16.156 \pm 0.482$ & $12.815 \pm 0.495$ & $8.816 \pm 0.563$ \\
3 & 0.027 & -19.70 & -19.29 & $19.186 \pm 0.315$ & $20.068 \pm 0.654$ & $18.336 \pm 0.551$ & $11.384 \pm 0.282$ & $10.401 \pm 0.848$ \\
\hline
\end{tabular}

* The unit for MITH is $\mathrm{mm}^{5}$, and the unit for $\left|S_{11}\right|$ and SLL is dB.

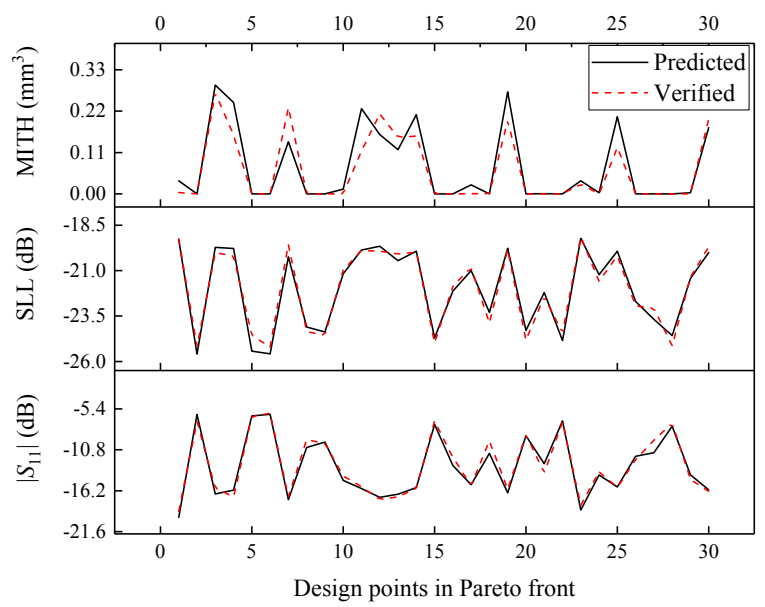

Fig. 7. Predicted and validated Pareto front for the SMAA with $i=4$.

TABLE III

COMPUTATION TIME FOR THE SMAA DESIGN.

\begin{tabular}{ccc}
\hline Operation & Time & Remark \\
\hline EM simulation & 59.40 hours & 1529 evals \\
\hline Optimization & 70.96 hours & $\begin{array}{c}0.22 \text { billion } \\
\text { surrogate evals }\end{array}$ \\
\hline Surrogate model training & 2.51 hours & 24225 times \\
\hline Overall & 133.07 hours & \\
\hline
\end{tabular}

based on the available dataset for antenna responses. In the outer loop, new design points are sampled around the calculated Pareto front, and the surrogate models for antenna responses are updated online. For each performance objective, single-objective optimization is carried out to optimize each individual objective using the surrogate models; the optimized results are then used to seed the multiobjective optimization procedure, thereby enhancing the performance [19].

In the inner loop, the predicted antenna performance responses $\mathbf{P}_{\text {pre }, i, j}$ are verified using EM simulations. The surrogate models for the antenna performance are then updated. The corresponding MITH of $\mathbf{P}_{\text {pre }, i, j}$ is also updated, with the obtained Pareto front named $\mathbf{P}_{\mathrm{val1}, i, j}$. The termination criterion can be set as the maximum number of iterations of the inner loop $J$ or a limit on the RMSE. If the termination criterion is fulfilled, then the algorithm shifts to the outer loop, in which a number of design points $N_{\text {sam }}$ are randomly sampled around every design point in the Pareto front with verified antenna responses $\mathbf{P}_{\text {val1 }, i, j}$ within circular regions of radii $k_{\text {sam }} \times D_{\text {sam }}$ and at the midpoint between each design point and the corresponding WCP point, where $D_{\text {sam }}$ is the distance between these two points and $k_{\text {sam }}$ is the coefficient. Local surrogate models are updated, so improved accuracy is achieved when predicting the antenna responses around $\mathbf{P}_{\mathrm{val}, i}$. Surrogate models for the parameter tolerances are then updated based on the updated local surrogate models for the antenna responses using MITH searching and WCA. The parameter tolerances for the obtained Pareto front with verified antenna responses are then recalculated based on the updated surrogate models. The Pareto front obtained here is $\mathbf{P}_{\mathrm{val} 2, i}$, in which the parameter tolerance can be regarded as a verified MITH based on the accurate local surrogate models.

Based on the verified results for both the antenna responses and the parameter tolerances, Pareto front $\mathbf{P}_{\text {upd }, i}$ is then obtained based on the entire dataset, which is regarded as the final HF results in one outer loop. Whereas $\mathbf{P}_{\mathrm{val}, i}$ gives a relatively accurate Pareto front prediction, the final Pareto front should be further updated due to the presence of inevitable prediction bias. The Pareto front obtained here is $\mathbf{P}_{\text {upd, } i}$, which can be utilized for tradeoff purposes in the final design. The termination criterion for the outer loop can be set as the maximum number of iterations of the outer loop $I$. By introducing a two-level nested loop, the surrogate models for the design points on and around the predicted Pareto front are built and updated within the robust design framework. The Pareto front is verified and updated to achieve better accuracy. One antenna design is shown here to verify the proposed ML-MLAO algorithm.

The series-fed microstrip antenna array (SMAA) shown in Fig. 5 is analyzed and optimized based on the proposed optimization and robust design method. The SMAA is designed on the ground plane with a Rogers 5880 substrate having dimensions of $400 \times 30 \times 1.5 \mathrm{~mm}^{3}$ 
and a relative permittivity of $\epsilon_{r}=2.3$. The antenna is designed for $5.8 \mathrm{GHz}$ with 10 series-fed microstrip antenna elements of symmetric structure and shortened at the end. The distance between different elements, the width of the antenna elements and the width of the microstrip line are constant at $g_{0}=17 \mathrm{~mm}, m_{0}=2 \mathrm{~mm}$ and $w_{1}=16.4 \mathrm{~mm}$. The output tolerance is set with a maximum $\left|S_{11}\right|$ better than $-14 \mathrm{~dB}$ and an SLL better than $-18 \mathrm{~dB}$. The design vector is $\boldsymbol{x}=\left[l_{1}, l_{2}, l_{3}, l_{4}, l_{5}\right]^{\mathrm{T}}$, and the design space is defined by the center vector $\boldsymbol{x}^{0}=[20.5,20,18,11.5,9]^{\mathrm{T}} \mathrm{mm}$ with a variable range of $\boldsymbol{x}^{0} \pm \delta$, where $\delta=[1.5,2,3,2.5,3]^{\mathrm{T}} \mathrm{mm}$. The WCA design space should be larger than the design space for design cases in which the WCP points appear near the boundaries; hence, the WCA design space is set as $\boldsymbol{x}^{0} \pm \delta_{\mathrm{w}}$, where $\delta_{\mathrm{w}}=[2,2.5,3.5,3,3.5]^{\mathrm{T}}$ $\mathrm{mm}$. The optimization and SA phases are performed in turn with $J=1, I=4, k_{\text {sam }}=1.2, N_{\text {sam }}=30, N_{\mathrm{u}}=5, N_{\mathrm{r}}=5$ and $N_{\text {iter }}=5$. The design objectives are the reflection coefficient $\left|S_{11}\right|$ and SLL at the designated frequency point and the MITH. The Pareto front for the designed SMAA after four iterations is shown in Fig. 6, with $\mathbf{P}_{\text {pre }, 4}, \mathbf{P}_{\text {val2,4 }}$ and $\mathbf{P}_{\text {upd,4 }}$ presented. The predicted and verified antenna responses and tolerances are shown in Fig. 6. The algorithm gives a close prediction for both antenna responses and the MITH.

Three design points on the obtained final Pareto front are highlighted, and their corresponding dimensions and performance values are given in Table II. A clear tradeoff between the antenna performance and robustness can be ascertained. The data in Table II demonstrate that while Design 3 has the best $\left|S_{11}\right|$ performance, Design 2 has comparable $\left|S_{11}\right|$ performance and much better SLL performance. Compared with Design 2, Design 1 suffers from worse $\left|S_{11}\right|$ and SLL performance but has much better parameter tolerances, which is more suitable when robustness is considered in the final fabrication process. A summary of the computation time for the SA process of the SMAA design is listed in Table III. The computation time has been largely reduced by using surrogate models for not only the antenna responses $\left|S_{11}\right|$ and SLL but also the WCP points and MITHs. A total of 420 MITH searches are conducted, where each implementation takes approximately $10 \mathrm{~min}$ of computation time, and the corresponding MITHs are predicted 50,600 times, which means that the overall computation time increases to approximately $8587 \mathrm{~h}$ if no surrogate models for the MITHs are used. A total of 14,678 WCAs are conducted, where each implementation takes approximately $10.0 \mathrm{~s}$ of computation time, and approximately 65 million predictions are made for the WCP, with 15,4857 predictions for the WCP in one MITH search process. The entire computation time can reach approximately 21.8 million $\mathrm{h}$ (approximately 2482 years) if no surrogate models for the WCP and MITH are used. A computation time of $139.9 \mathrm{~s}$ is needed to conduct the HFSS simulation of the SMAA. In one WCA process, approximately 5445 function calls for the $\left|S_{11}\right|$ and SLL performance are needed. If no surrogate models are used for the WCP, MITH, $\left|S_{11}\right|$ and SLL, then the computation time will increase to approximately 189 million years. It is worth noting that all computation times listed above, except the time using all surrogate models, are roughly estimated based on the evaluation times of the surrogate models; these estimates are calculated only to show how the application of surrogate models for different design objectives can help largely reduce the entire computation time.

\section{CONCLUSiON}

An ML-MLAO method has been proposed to achieve reliable and efficient robust design for antenna and array applications. By introducing MLAO algorithms to different layers of the robust design process, rapid and trustworthy WCA, MITH searching and robust optimization have been achieved. The heavy computational loads for global searching, EM simulation and tolerance analysis have been greatly reduced by exploiting prior knowledge regarding the correlations between the antenna parameters and responses, WCP and MITH. This increased efficiency has been utilized to make predictions in different design layers, largely accelerating the whole robust design process. Moreover, the surrogate models for the aforementioned design objectives are updated online following the MLMLAO algorithm. Examples including array synthesis and antenna design have been described to verify the efficiency and reliability of the proposed method.

\section{REFERENCES}

[1] K. Deb and H. Gupta, "Introducing robustness in multi-objective optimization," Evol. Comput., vol. 14, no. 4, pp. 463-494, 2006.

[2] J. A. Easum, J. Nagar, P. L. Werner, and D. H. Werner, "Efficient multiobjective antenna optimization with tolerance analysis through the use of surrogate models," IEEE Trans. Antennas Propag., vol. 66, no. 12, pp. 6706-6715, 2018.

[3] A. Kouassi, N. Nguyen-Trong, T. Kaufmann, S. Lalléchère, P. Bonnet, and C. Fumeaux, "Reliability-aware optimization of a wideband antenna," IEEE Trans. Antennas Propag., vol. 64, no. 2, pp. 450-460, 2015.

[4] B. Zhang and Y. Rahmat-Samii, "Robust optimization with worst case sensitivity analysis applied to array synthesis and antenna designs," IEEE Trans. Antennas Propag., vol. 66, no. 1, pp. 160-171, 2018.

[5] C. Hu, S. Zeng, Y. Jiang, J. Sun, Y. Sun, and S. Gao, "A robust technique without additional computational cost in evolutionary antenna optimization," IEEE Trans. Antennas Propag., vol. 67, no. 4, pp. $2252-$ 2259, 2019.

[6] J. Lee, Y. Lee, and H. Kim, "Decision of error tolerance in array element by the monte carlo method," IEEE Trans. Antennas Propag., vol. 53, no. 4, pp. 1325-1331, 2005.

[7] B. Xia, Z. Ren, and C.-S. Koh, "Utilizing kriging surrogate models for multi-objective robust optimization of electromagnetic devices," IEEE Trans. Magn., vol. 50, no. 2, pp. 693-696, 2014.

[8] H. Schjaer-Jacobsen and K. Madsen, "Algorithms for worst-case tolerance optimization," IEEE Trans. Circuits Syst., vol. 26, no. 9, pp. 775-783, 1979

[9] B. Liu, H. Aliakbarian, Z. Ma, G. A. Vandenbosch, G. Gielen, and P. Excell, "An efficient method for antenna design optimization based on evolutionary computation and machine learning techniques," IEEE Trans. Antennas Propag., vol. 62, no. 1, pp. 7-18, 2014.

[10] J. P. Jacobs and S. Koziel, "Two-stage framework for efficient gaussian process modeling of antenna input characteristics," IEEE Trans. Antennas Propag., vol. 62, no. 2, pp. 706-713, 2014.

[11] N. Leszczynska, I. Couckuyt, T. Dhaene, and M. Mrozowski, "Lowcost surrogate models for microwave filters," IEEE Microw. and Wireless Components Lett., vol. 26, no. 12, pp. 969-971, 2016.

[12] W. Lyu, P. Xue, F. Yang, C. Yan, Z. Hong, X. Zeng, and D. Zhou, "An efficient bayesian optimization approach for automated optimization of analog circuits," IEEE Trans. Circuits Syst. I, Reg. Papers, vol. 65, no. 6, pp. 1954-1967, 2018.

[13] S. Koziel and S. Ogurtsov, "Multi-objective design of antennas using variable-fidelity simulations and surrogate models," IEEE Trans. Antennas Propag., vol. 61, no. 12, pp. 5931-5939, 2013.

[14] Y. Kim, S. Keely, J. Ghosh, and H. Ling, "Application of artificial neural networks to broadband antenna design based on a parametric frequency model," IEEE Trans. Antennas Propag., vol. 55, no. 3, pp. 669-674, 2007.

[15] Q. Wu, H. Wang, and W. Hong, "Multi-stage collaborative machine learning and its application to antenna modeling and optimization," IEEE Trans. Antennas Propag., vol. 68, no. 5, pp. 3397-3409, 2020.

[16] Q. Wu, Y. Cao, H. Wang, and W. Hong, "Machine-learning-assisted optimization and its application to antenna designs: opportunities and challenges," China Commun., vol. 17, no. 4, pp. 152-164, 2020.

[17] C. E. Rasmussen, "Gaussian processes in machine learning," in Summer School on Machine Learning. Springer, 2003, pp. 63-71.

[18] Y. Cao, Q. Wu, H. Wang, and W. Hong, "Design of broadband reflectarray antenna using machine-learning-assisted optimization method," in 2019 International Symposium on Antennas and Propagation (ISAP), 2019, pp. 1-3.

[19] T. Friedrich and M. Wagner, "Seeding the initial population of multiobjective evolutionary algorithms: a computational study," Applied Soft Computing, vol. 33, pp. 223-230, 2015. 